\title{
Kinematics Modeling and Analysis of MOTOMAN-HP20 Robot
}

\author{
Jiyou Fei, Chen Huang \\ School of Mechanical Engineering, Dalian Jiaotong University, Dalian, China \\ huagnchen054@163.com
}

Keywords: Rotation matrix; forward kinematics; inverse kinematics; robot workspace

\begin{abstract}
The improved kinematics method based on the orthogonality of rotation matrix and matrix block is presented for MOTOMAN - HP20 robot to improve the computing speed of the robot kinematics.Firstly, according to the robot structure, the forward kinematics model is established with $\mathrm{D}-\mathrm{H}$ method. And for the inverse kinematics, the separation method of position and orientation matrix is used to transform complex matrix equation into eight algebraic equations to improve the computing speed. For extraneous roots, the optimal solution is confirmed by selecting the closest one to the current joint angle with the solving order directly, instead of computing all the roots. Finally, the workspace is obstained by the simulation and the kinematics analysis method is proved to be efficient.
\end{abstract}

\section{Introduction}

For the kinematics analysis, the forward kinematics equation is generally established by D-H kinematics parameters, but the inverse kinematics is more complicated, and there are some popular methods, e.g. the matrix transformation method based on D-H parameters [2]. Matrix transformation is widely applied, but the method needs more homogeneous transformation matrix inversion and repeatedly multiplication by inverse matrix. Another method is geometric method for the robot structure [3]. Although it has the advantage of fast operation speed, it is limited by the structure of the robot. Screw transformation and quaternion method [4] is more complicated. Neural network [5], genetic algorithm [6] etc. also have been used to the inverse kinematics. But the stability and precision are still unable to meet the requirements of real-time control. So some modified methods have been proposed to improve the operation rate of matrix transformation method [7-8]. The vector operation of a nonlinear equation [9] was also proposed to solve the inverse kinematics problem.

For MOTOMAN-HP20 robot, an inverse kinematic method is presented to avoid inverse computation of large-scale matrices based on the orthogonality theory of the rotation matrix, homogeneous matrix is decomposed into rotation matrix and position matrix, the inversion for the matrix is transferred into transposition operation, improving the solving efficiency. And according to the principle of "minimum distance" for each joint angle, the optimal solution of the inverse kinematics is selected to avoid solving extraneous roots. Finally, the robot workspace was obtained by simulation, and the kinematics method is verified to be correct.

\section{Forward kinematics analysis}

MOTOMAN - HP20 is six degrees of freedom (6 DOF) serial chain robot with a spherical wrist. The coordinate system is shown in Fig.1.

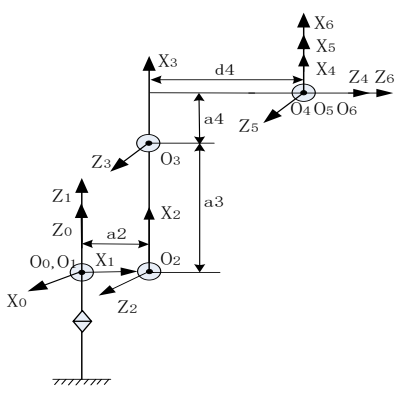

Fig.1 The coordinate system of MOTOMAN-HP20 
Robot D-H parameters are shown from Table 1.

Table $1 \mathrm{D}-\mathrm{H}$ parameters table

\begin{tabular}{cccccc}
\hline $\begin{array}{l}\text { Join } \\
\mathrm{t}\end{array}$ & $\theta\left(^{\circ}\right)$ & $d(\mathrm{~mm})$ & $\alpha\left(^{\circ}\right)$ & $a(\mathrm{~mm})$ & $\theta$ range $\left(^{\circ}\right.$ \\
\hline 1 & $\theta_{1}$ & 0 & 0 & 0 & $-180 \sim 180$ \\
2 & $\theta_{2}$ & 0 & 90 & $a_{2}=150$ & $-110 \sim 155$ \\
3 & $\theta_{3}$ & 0 & 0 & $a_{3}=760$ & $-165 \sim 255$ \\
4 & $\theta_{4}$ & $d_{4}=795$ & 90 & $a_{4}=140$ & $-200 \sim 200$ \\
5 & $\theta_{5}$ & 0 & -90 & 0 & $-50 \sim 230$ \\
6 & $\theta_{6}$ & 0 & 90 & 0 & $-360 \sim 360$ \\
\hline
\end{tabular}

Use the homogeneous matrix of D-H to describe the coordinate transformation matrix of two adjacent links, the matrix [9] can be expressed as

$$
{ }_{i}^{i-1} T=\left[\begin{array}{cccc}
\cos \theta_{i} & -\sin \theta_{i} & 0 & a_{i} \\
\sin \theta_{i} \cos \alpha_{i} & \cos \theta_{i} \cos \alpha_{i} & -\sin \alpha_{i} & -\sin \alpha_{i} d_{i} \\
\sin \theta_{i} \sin \alpha_{i} & \cos \theta_{i} \sin \alpha_{i} & \cos \alpha_{i} & \cos \alpha_{i} d_{i} \\
0 & 0 & 0 & 1
\end{array}\right]=\left[\begin{array}{cc}
R_{i} & P_{i} \\
0 & 1
\end{array}\right]
$$

where $a_{i}$ is the link length, $d_{i}$ is the distance between adjacent link, $R_{i}$ is $3 \times 3$ rotation matrix:

$$
R_{i}=\left[\begin{array}{ccc}
\cos \theta_{i} & -\sin \theta_{i} & 0 \\
\sin \theta_{i} \cos \alpha_{i} & \cos \theta_{i} \cos \alpha_{i} & -\sin \alpha_{i} \\
\sin \theta_{i} \sin \alpha_{i} & \cos \theta \sin \alpha_{i} & \cos \alpha_{i}
\end{array}\right]
$$

$P_{i}$ is $3 \times 1$ position sub-matrix as

$$
P_{i}=\left[a_{i} ;-d_{i} \sin \alpha_{i} ; d_{i} \cos \alpha_{i}\right]
$$

Then the kinematics equation can be

$$
{ }_{6}^{0} T={ }_{1}^{0} T_{2}^{1} T_{3}^{2} T_{4}^{3} T_{5}^{4} T_{6}^{5} T=\left[\begin{array}{cc}
R & P \\
0 & 1
\end{array}\right]=\left[\begin{array}{cccc}
n_{x} & o_{x} & a_{x} & p_{x} \\
n_{y} & o_{y} & a_{y} & p_{y} \\
n_{z} & o_{z} & a_{z} & p_{z} \\
0 & 0 & 0 & 1
\end{array}\right]
$$

where ${ }_{6}^{0} T$ is the pose matrix; $\left[\begin{array}{lll}p_{x} & p_{y} & p_{z}\end{array}\right]^{T}$ is the position vector, $\left[n_{x} n_{y} n_{z}\right]^{T},\left[\begin{array}{lll}o_{x} & o_{y} & o_{z}\end{array}\right]^{T},\left[\begin{array}{lll}a_{x} & a_{y} & a_{z}\end{array}\right]^{T}$ are respectively orientation vector of the coordinate system of the end operator relative to the $x, y$ and $z$ axis of the reference coordinate system.

By the D-H parameters in Table.1 with the Eq.(1), the homogeneous transformation matrix between the adjacent links can be written as

$$
\begin{aligned}
& { }_{1}^{0} T=\left[\begin{array}{cccc}
\cos \theta_{1} & -\sin \theta_{1} & 0 & 0 \\
\sin \theta_{1} & \cos \theta_{1} & 0 & 0 \\
0 & 0 & 1 & 0 \\
0 & 0 & 0 & 1
\end{array}\right]{ }_{2}^{1} T=\left[\begin{array}{cccc}
\cos \theta_{2} & -\sin \theta_{2} & 0 & 150 \\
0 & 0 & -1 & 0 \\
\sin \theta_{2} & \cos \theta_{2} & 0 & 0 \\
0 & 0 & 0 & 1
\end{array}\right] \quad{ }_{3}^{2} T=\left[\begin{array}{cccc}
\cos \theta_{3} & -\sin \theta_{3} & 0 & 760 \\
\sin \theta_{3} & \cos \theta_{3} & 0 & 0 \\
0 & 0 & 1 & 0 \\
0 & 0 & 0 & 1
\end{array}\right] \\
& { }_{4}^{3} T=\left[\begin{array}{cccc}
\cos \theta_{4} & -\sin \theta_{4} & 0 & 140 \\
0 & 0 & -1 & -795 \\
\sin \theta_{4} & \cos \theta_{4} & 0 & 0 \\
0 & 0 & 0 & 1
\end{array}\right] \quad{ }_{5}^{4} T=\left[\begin{array}{cccc}
\cos \theta_{5} & -\sin \theta_{5} & 0 & 0 \\
0 & 0 & 1 & 0 \\
-\sin \theta_{5} & -\cos \theta_{5} & 0 & 0 \\
0 & 0 & 0 & 1
\end{array}\right] \quad{ }_{6}^{5} T=\left[\begin{array}{cccc}
\cos \theta_{6} & -\sin \theta_{6} & 0 & 0 \\
0 & 0 & -1 & 0 \\
\sin \theta_{6} & \cos \theta_{6} & 0 & 0 \\
0 & 0 & 0 & 1
\end{array}\right]
\end{aligned}
$$

Then $n, o, a, p$ can be derived in $x, y, z$ index.

\section{Inverse kinematics solution}

\subsection{Inverse kinematics method}

The inverse kinematics of the robot is the process to compute joint angle of each link based on given pose of end-effector operator, which plays an important role in the robot control system. The condition that the robot existing a closed form solution of inverse kinematics is to meet one of two 
conditions of Pieper criteris [11] for the robot's mechanical structure: (1) The axises of three adjacent joint need to intersect to one point; (2) The axises of three adjacent joint are parallel to each other. The mechanical structure is designed with the axes of the three adjacent joint intersect, so the closed-form solution exists. Transformation matrix with the two adjacent link as Eq.(1) is divided into rotation matrix and position matrix, with inverse rule of partitioned matrix the rotation sub-matrix is orthogonal matrix, it meets $R_{i}^{-1}=R_{i}^{T}$. Thus,

$$
\left({ }_{i}^{i-1} T\right)^{-1}=\left[\begin{array}{cc}
R_{i}^{T} & -R_{i}^{T} P_{i} \\
0 & 1
\end{array}\right](i=1,2, \cdots, 6)
$$

Therefore, inversion compution of the rotation matrix is converted to compute transpose. Thereby, the amount of calculation significantly is reduced. To find the inverse kinematics solution, link transformation equation can be rewritten as

$$
\left({ }_{2}^{1} T\right)^{-1}\left({ }_{1}^{0} T\right)^{-1}{ }_{6}^{0} T\left({ }_{6}^{5} T\right)^{-1}={ }_{3}^{2} T_{4}^{3} T{ }_{5}^{4} T
$$

The left and right matrixes of Eq.(6) are

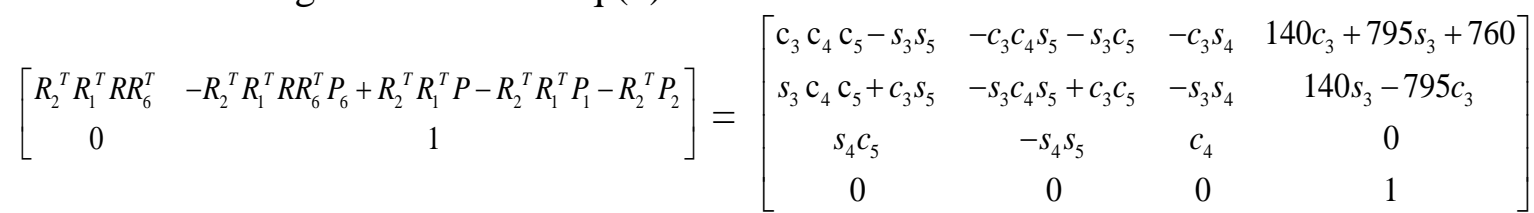

Considering the structure characteristics, the first three joint angles $\left(\theta_{1}, \theta_{2} 、 \theta_{3}\right)$ are used to decide the position of the end-effector, and then the last three joint angles $\left(\theta_{4}, \theta_{5}, \theta_{6}\right)$ determine the oriention of the end-effector, so the solution of the inverse kinematics can be decomposed into the position and posture matrixes. For the left and right sides of Eq.(6) are equal, and the equations for the first three joint angles can be derived by the corresponding element in the position sub-matrices, and by the posture matrix, five equations for the last three joint angles can be obtained. Suppose $c_{i}=\cos \theta_{i}$ and $s_{i}=\sin \theta_{i}$, then the equations are as follows:

$$
\left\{\begin{array}{l}
s_{1} p_{x}-c_{1} p_{y}=0 \\
c_{2}\left(p_{x} c_{1}+p_{y} s_{1}-150\right)+p_{z} s_{2}=795 s_{3}+140 c_{3}+760 \\
-s_{2}\left(p_{x} c_{1}+p_{y} s_{1}-150\right)+p_{z} c_{2}=140 s_{3}-795 c_{3} \\
-c_{2}\left(c_{1} a_{x}+s_{1} a_{y}\right)-s_{2} a_{z}=-c_{3} c_{4} s_{5}-s_{3} c_{5} \\
s_{2}\left(c_{1} a_{x}+s_{1} a_{y}\right)-c_{2} a_{z}=-s_{3} c_{4} s_{5}+c_{3} c_{5} \\
-s_{1} a_{x}+c_{1} a_{y}=-s_{4} s_{5} \\
\left(c_{1} n_{y}-s_{1} n_{x}\right) c_{6}+\left(s_{1} o_{x}-c_{1} o_{y}\right) s_{6}=-s_{4} c_{5} \\
\left(c_{1} n_{y}-s_{1} n_{x}\right) s_{6}-\left(s_{1} o_{x}-c_{1} o_{y}\right) c_{6}=-c_{4}
\end{array}\right.
$$

By solving the above eight equations, various joint angles can be shown as follows: $\theta_{1}=A \tan 2\left(\mathrm{p}_{y}, \mathrm{p}_{x}\right)$ or $A \tan 2\left(-\mathrm{p}_{y},-\mathrm{p}_{x}\right) ; \theta_{2}=A \tan 2\left(h_{3} p_{z}-h_{1} h_{4}, h_{1} h_{3}+p_{z} h_{4}\right)$;

$\theta_{3}=A \tan 2\left(h_{2}, \sqrt{1-h_{2}^{2}}\right)-A \tan 2(140,795)$ or $A \tan 2\left(h_{2},-\sqrt{1-h_{2}^{2}}\right)-A \tan 2(140,795)$;

$\theta_{4}=A \tan 2\left(\mathrm{c}_{1} \mathrm{a}_{y}-\mathrm{s}_{1} \mathrm{a}_{x}, \mathrm{a}_{z} \mathrm{~s}_{23}+\mathrm{c}_{23}\left(\mathrm{c}_{1} \mathrm{a}_{x}+\mathrm{s}_{1} \mathrm{a}_{y}\right)\right) ; \theta_{5}=A \tan 2\left(\sqrt{1-g^{2}}, g\right)$ or $A \tan 2\left(-\sqrt{1-g^{2}}, g\right)$;

$\theta_{6}=A \tan 2\left(s_{4} c_{5}\left(s_{1} n_{x}-c_{1} n_{y}\right), s_{4} c_{5} c_{4}-s_{4} c_{5}\left(s_{1} o_{x}-c_{1} o_{y}\right)\right)$.

where $h_{1}=p_{x} c_{1}+p_{y} s_{1}-150, h_{2}=\left(h_{1}^{2}+p_{z}^{2}-140^{2}-795^{2}-760^{2}\right) / 1520 \sqrt{140^{2}+795^{2}}, h_{3}=140 c_{3}+795 s_{3}+760$, $h_{4}=140 s_{3}-795 c_{3}, g=s_{23}\left(c_{1} a_{x}+s_{1} a_{y}\right)-c_{23} a_{z}$.

\subsection{Choosing method of the optimal solution}

For any given end-effector pose, there are eight groups of inverse solution. But only a set of definite solution is needed, so choose the closest solution to the last angle from multiple sets of inverse solution within the scope of the joint movement. In general, the optimal solution of inverse is selected after the inverse solutions of all the joint angles are calculated, and the optimal set among all the inverse solutions is that the sum of all the joint angles is smallest. It can be expressed as

$$
\min S=\min \sum_{i=1}^{6}\left|\theta_{i}-\theta_{i b}\right|
$$

But this method requires to compute all the solutions, and then to determine a set of the optimal 
solution. To reduce the motion time and ensure the lest change of the joint angle from the current pose to the next pose, only make sure the change value of every joint angle is minimum, and the change sum of all angles is minimum. Therefore in the process of computing the joint angles of inverse kinematics, for the joint angles that exist multiple solutions, firstly, to estimate whether the value of these joint angles is within the allowed scope, then by $\min \left|\theta_{i}-\theta_{i b}\right|$ to determine the most optimal solution, using the optimal solution to calculate the next joint angle and determine the minimum value of the next joint angle by the same way.

\section{Simulation and verification of kinematics}

\subsection{Simulation of the robot workspace}

Workspace is an important kinematics indexes for assessing the robot ability to work. The most common method to calculate the workspace is the monte-carlo method [11], the cycle strategy is used to calculate the robot workspace in this paper. The position vector of robot end-effector is given in Eq.(14), the scope of the workspace is determined by the position vector, according to $p_{x} 、 p_{y}$ 、 $p_{z}$ and the scope of the robot joint angle to determine the robot workspace. The specific steps are:

(1) The position vector: $p_{x}=140 c_{1} c_{23}+795 c_{1} s_{23}+760 c_{1} c_{2}+150 c_{1}, p_{y}=140 s_{1} c_{23}+795 s_{1} s_{23}+760 s_{1} c_{2}+150 s_{1}$, and $p_{z}=140 s_{23}-795 c_{23}+760 s_{2}$.

(2) The range of the joint angle is as follows:

$$
\left(-\pi \leq \theta_{1} \leq \pi, \quad-\frac{11}{18} \pi \leq \theta_{2} \leq \frac{31}{36} \pi,-\frac{11}{12} \pi \leq \theta_{3} \leq \frac{17}{12} \pi,-\frac{10}{9} \pi \leq \theta_{4} \leq \frac{10}{9} \pi,-\frac{5}{18} \pi \leq \theta_{5} \leq \frac{23}{18} \pi,-2 \pi \leq \theta_{6} \leq 2 \pi\right)
$$

all the end-effector coordinates within the change scope of the joint angle can be obtained.

(3) The workspace result is shown in Fig.2.

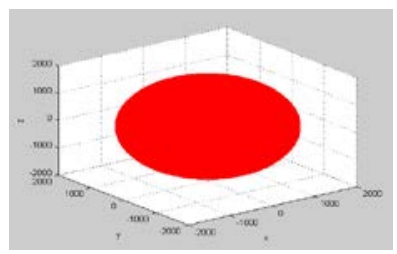

(a) XYZ 3D workspace

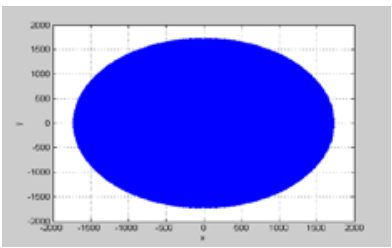

(b) XOY 2D workspace Fig.2 The robot workspace

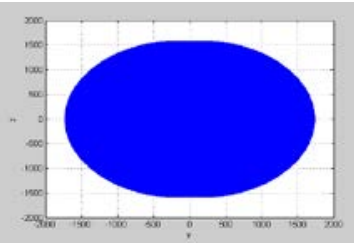

(c) YOZ 2D workspace

\subsection{Verification of the robot kinematics}

Firstly, a motion trajectory is given. The trajectory within 0s to 50s: $p_{x}=\sin (t) ; p_{y}=10 \times(t-\sin (t))+670$; $p_{z}=10 \times(1-\cos (t))+415$ from Fig.3(a). Secondly, keep the orientation of the robot end-effector as $R=[01$ 0; $001 ; 10$ ]. According to solving inverse kinematics of MOTOMAN-HP20, the changes of the six joint angles are shown in Fig.4. By the forward kinematics equation, the position vector can be obtained. The simulation trajectory of the manipulator is shown in Fig.3(b). Finally, comparing with the result of Fig.3(a) and Fig.3(b), the trajectory curve by the forward kinematics equation is the same as the curve space given in Fig.3(a), the correctness of the forward and inverse kinematics solution can be verified in this work.
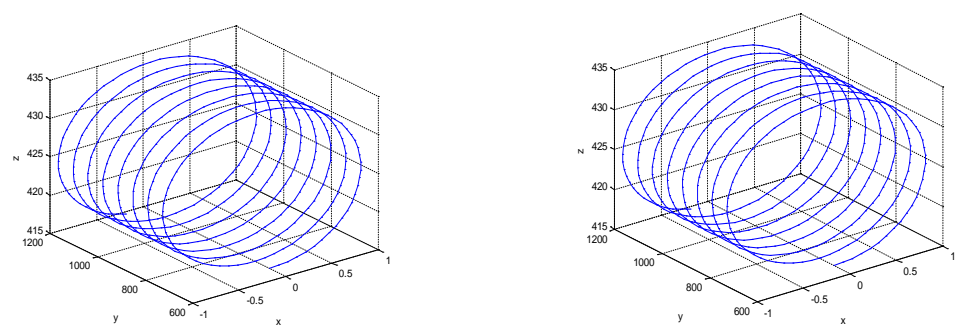

Fig.3 (a) The given space spiral curve; (b) The trajactory of the forward kinematics 


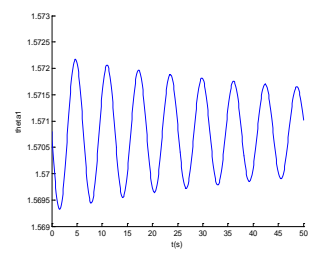

(a) the change curve of angle $\theta_{1}$

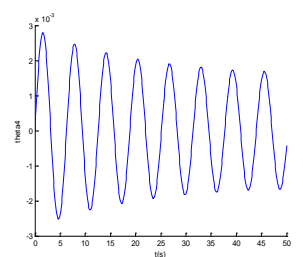

(d) the change curse of angle $\theta_{4}$
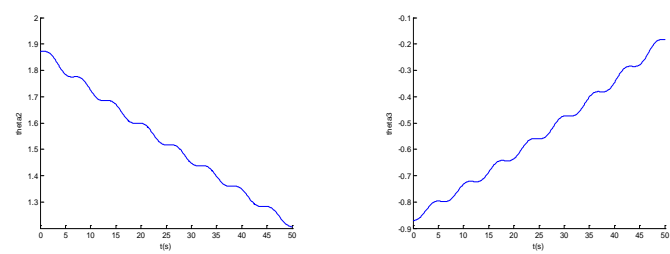

(b) the change curve of angle $\theta_{2}$

(c) the change curve of angle $\theta_{3}$
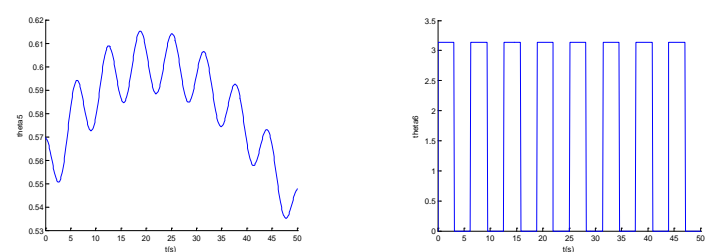

(f) the change curse of angle $\theta_{6}$

\section{Summary}

The kinematics of MOTOMAN - HP20 robot was studied in the paper, and a kind of method about the kinematics analysis based on rotation matrix orthogonality and matrix block thought is put forward. By the simulation, the robot workspace is calculated, and the correctness of the forward kinematics solution and inverse kinematics model is given to provide the theoretical foundation for the research of robot control and trajectory planning optimization. This method can also be used to provide the kinematics reference for other industrial robot with six-degree- of-freedom.

\section{References}

[1]. Liu SG, Zhu SQ, et al. Research on real-time inverse kinematics algorithms for 6R robots. Control Theory \& Applications, 2008, Vol.25 (2008) No.6, p.1037-1041.

[2]. Wang QJ, Du JJ. A new solution for inverse kinematics problems of MOTOMAN robot. Journal of Harbin Institute of Technology, vol.3 (2010), p. 451-454.

[3]. Fu KS, Gonzalez RC, Lee CSG. Robotics: control, sensing, vision, and intelligence. McGraw-Hill Education, 1987.

[4]. Lv SZ, et al. Solution of Screw Equation for Inverse Kinematics of 6R Robot Based on Wu's Method. Journal of Mechanical Engineering, Vol.46 (2010) No.17, p. 35-41.

[5]. Mayorga RK, Sanongboon P. A radial basis function net-work approach for geometrically bounded manipulator inverse kinematics computation, IEEE International Conference on Intelligent Robots and Systems. 2003, p. 3564-3569.

[6]. Huang W, Tan S, Li X. Inverse Kinematics of Com-pliant Manipulator Based on the Immune Genetic Algo-rithm, Fourth International Conference on Natural Computation. IEEE Computer Society, 2008, p. 390-394.

[7]. Manocha D, Canny JF. Efficient inverse kinematics for general 6R manipulators. Robotics \& Automation IEEE Transactions on, Vol.5 (1994) No.10, p. 648-657.

[8]. Liu H. Real-time Inverse Kinematics Algorithm Based on Vector Dot Product. Transactions of the Chi-nese Society for Agricultural Machinery, 2009, Vol.40 (2009) No.6, p. 212-218.

[9]. Cheng YL, Zhu SQ, Liu SG. Inverse Kinematics of 6R Robots Based on the Orthogonal Character of Rotation Sub-matrix. Robot, Vol.30 (2008) No.2, p. 160-164.

[10]. Siciliano B, Khatib O. Springer Handbook of Robotics. Springer, 2008.

[11]. Feng Y, Wang YN, Yu HS. Workspace boundary extraction of de-icing robot based on Monte Carlo method. Control Theory \& Applications, Vol.27 (2010) No.7, p. 891-896. 